\title{
Effects of Anesthesia on Effective Connectivity in the Brain
}

\author{
Xinyu Xu' ${ }^{1}$, Guolin Wang ${ }^{2 *}$, Xin Tian ${ }^{1 *}$ \\ ${ }^{1}$ Laboratory of Neurobiology in Medicine, School of Biomedical Engineering, Tianjin Medical University, Tianjin, \\ China \\ ${ }^{2}$ Department of Anesthesiology, Tianjin Medical University General Hospital, Tianjin Institute of Anesthesiology, \\ Tianjin, China \\ Email: wang guolin@hotmail.com, ${ }^{*}$ tianx@tmu.edu.cn
}

Received 21 February 2015; accepted 25 April 2015; published 30 April 2015

Copyright (C) 2015 by authors and Scientific Research Publishing Inc.

This work is licensed under the Creative Commons Attribution International License (CC BY).

http://creativecommons.org/licenses/by/4.0/

(c) (i) Open Access

\begin{abstract}
The brain constitutes a formidably complicated structural network. There are three main types of connectivity used to describe neuronal networks, which reflect three parallel levels of investigation: anatomical connectivity, functional connectivity and effective connectivity. Effective connectivity indicates the direct influence that a node exerts on another, and in the context of neuronal circuits, a causal relationship between the activities of two nodes. Since its definition, effective connectivity analysis has been used to describe causal relationship across multiple spatial scales in PET imaging, fMRI, electroencephalography (EEG) and magnetoencephalography (MEG), singleunit, and local field potential. There are diverse literatures which probe the anesthetized state using effective connectivity analysis over the past two decades. The examination of effective connectivity in the anesthetized state is of relevance to both anesthesiologists and neuroscientists, as it has the potential to elucidate still unclear mechanisms of anesthesia while offering insight into intrinsic functional activity in the brain. The present review attempts to examine, elucidate, and integrate the insight that effective connectivity analysis of the anesthetized state has generated thus far.
\end{abstract}

\section{Keywords}

Effective Connectivity, Anesthesia, Granger Causality, Consciousness

\section{Introduction}

Since the nineteenth century, we have known that the neuronal elements of the brain constitute a formidably

${ }^{*}$ Corresponding author. 
complicated structural network [1] [2]. Such networks are thought to provide the physiological basis for information processing and mental representations [3]-[6].

A network is defined by nodes, which are the elements of the network, and links (edges) connecting the nodes and defining structure. The nodes can be the neurons, neuronal ensembles and brain regions. There are three main types of connectivity used to describe neuronal networks, which reflect three parallel levels of investigation: anatomical connectivity, functional connectivity and effective connectivity. Anatomical connectivity indicates physical connections (i.e. chemical and electrical synapses, etc.) among nodes. Functional connectivity indicates a statistical dependence between the activities of two nodes without any assumption of the mechanism by which these relationships are mediated. Effective connectivity indicates the direct influence that a node exerts on another, and in the context of neuronal circuits, a causal relationship between the activities of two nodes [7]. Although effective connectivity can be also considered as one kind of functional connectivity, a distinction is usually made between functional connectivity and effective connectivity. Only the effective connectivity takes explicitly into consideration the existence of a causal link between two or more brain regions [8].

\section{How Is Effective Connectivity Determined?}

Effective connectivity research has branched into two formally distinct approaches: Dynamic Causal Modeling (DCM) [9], and Granger Causality (GC) [10].

The basic idea of DCM is that neural activity propagates through brain networks as in an input-state-output system, where causal interactions are mediated by unobservable neuronal dynamics. This multi-input multioutput neuronal model is augmented with a forward, or observation model that describes the mapping from neural activity to observed responses. Together neuronal and observation model comprise a full generative model that takes a particular form depending on the data modality.DCM was mainly used for analyzing fMRI.

From its introduction within econometrics, GC has been applied in neuroscience partly because it is simple to estimate, given (stationary stochastic) time series. GC also has some useful properties including a decomposition of causal influence by frequency and formulation in an 'ensemble' form, allowing evaluation of GC among multivariate sets of responses [11]. GC has provided useful descriptions of effective connectivity in EEG [12], MEG [13] and local field potentials [14]. But the application GC to fMRI is more controversial because of the slow dynamics and regional variability of the haemodynamic response to underlying neuronal activity [15] [16].

Other measures related to spectral GC have been proposed, such as the Directed Transfer Function (DTF) [17], the Partial Directed Coherence (PDC), which might be numerically and computationally advantageous when compared to any of the above measures [18]. Both DTF and PDC lend themselves to a multivariate approach and produce valid spectral causality estimates [19] [20].

The Phase Slope Index (PSI) is based on the idea that interactions between variables are not instantaneous and need time to propagate [21]. For example, two variables $\mathrm{X}$ and $\mathrm{Y}$, and provided that the propagation speed is constant, the phase difference between $\mathrm{X}$ and $\mathrm{Y}$ increases with frequency and the phase spectrum is expected to have a positive slope if $\mathrm{X}$ drives $\mathrm{Y}$ and a negative slope if $\mathrm{Y}$ drives $\mathrm{X}$. Another useful feature of PSI is insensitivity to mixtures of non-interacting sources which leads to a lower number of false positives when compared to GC.

Transfer Entropy (TE), based on the information theoretic context, was proposed [22] as a model-free, nonparametric measure of information transfer between two variables and has been applied to fMRI and EEG data [22]-[24]. The transfer entropy (TE) from the source to destination can be interpreted as the average mutual information between the previous state of the source and a future state of the destination, conditioned on past states of the destination.

\section{Why Study Effective Connectivity in Anesthesia?}

Despite advances in the understanding of anesthetic-receptor interactions for a variety of anesthetics, the specifics of why general anesthesia causes unconsciousness remain unclear [25]. Findings had indicated that different anesthetics produce the various components of anesthesia to varying degrees and with different dose responses [26]. Effective connectivity has provided one manner to assess the effects of various types and doses of anesthetics, for it provides not only the last effective networks to disappear prior to the onset of anesthetic-induced unconsciousness but also the first effective networks to reappear upon the recovery of consciousness [27]. More generally, effective studies provide a means of investigating the information flowing from one brain region to 
another at varying levels of anesthesia and, accordingly, the modulation of those networks by the various components of anesthesia.

\section{Studying Mechanisms of Anesthesia through Effective Connectivity}

Functional neuroimaging work on mechanisms of anesthesia has focused on understanding the specifics of the brain state corresponding to anesthesia-induced loss of consciousness (LOC). There are a number of hypotheses to consider. First and most simply, it may be possible to find regions of the brain in which activity is suppressed during AIU that wholly or partially account for LOC. A second possibility is that there may be impaired communication between specific brain regions that accounts for the LOC. Effective connectivity mapping is a highly appropriate means to search for this second type of finding.

1) Thalamus, thalamocortical connectivity, and consciousness

The thalamus plays a uniquely privileged role in the brain because all sensory information (except olfaction) passes through it. This unique location has made the thalamus a prime candidate to be the site of integration of percepts into a unified experience that we refer to as consciousness [28]. Many investigations have corroborated the concept that thalamic activity is significantly modulated in anesthesia. In 1993, Angel researched evoked potential recordings indicating that a blockade or disruption of sensory processing in the thalamus may be involved in LOC [29]. Soon after, Volkow and others showed that the depression of thalamic metabolism correlated with level of sedation induced by benzodiazepine lorazepam [30]. In 1999, Alkire and others demonstrated significant decreases in thalamic metabolism during halothane anesthesia, while Fiset $e t$ al. showed a correlation between thalamic activity and level of consciousness during propofol anesthesia [31] [32]. Those results gave more weight to the idea that thalamus switch on consciousness.

Thalamocortical networks play an important role in information integration during consciousness [33] [34]. How dose anesthetic affect on the thalamocortical connectivity? In order to find the answer, in 2012, Andrada and others recorded neuronal action potentials from cerebral cortex and thalamus before, during, and after infusion of either propofol or etomidate [35]. The result showed that neurons in the cortex and thalamus were similarly depressed by propofol and etomidate. Although Andrada work demonstrated anesthetic depression of neuronal activity likely contributes to anesthetic-induced unconsciousness, more work is needed to determine how anesthetic effects on thalamocortical interaction to produce unconsciousness. Then, Kim et al. used granger causality to investigate information flows in the thalamocortical network [36]. The result showed that $65 \%$ of the thalamocortical information flows were changed by anesthesia-induced LOC. Specifically, the information flows from the primary motor and somatosensory cortex to the ventral lateral thalamus before LOC whereas flows from the ventral lateral thalamus to the primary motor cortex and somatosensory cortex after LOC.

Based on the above research, suppression of thalamus by anesthetics may in fact follow in time the anesthetic suppression of cortical activity, suggesting an indirect role [37]. Thus, the thalamic effects of anesthesia are more likely to be consequential, secondary to the cortical effect of anesthetics.

2) Frontoparietal connectivity

Frontoparietal connectivity has been noted consistently as a neural correlate of consciousness [38]-[40].

To assess the effects of general anesthesia on frontoparietal connectivity, in 2005, Imas and others recorded event-related potentials from rat the primary visual cortex, parietal association and frontal cortex [41]. They used transfer entropy to examine the effect of two common volatile anesthetics, halothane and isoflurane, on information transfers between primary visual, parietal and frontal cortex. The result provided direct evidence that inhalational anesthetics impair frontal-posterior parietal cortex information transfer at high gamma frequencies in rats. In 2009, Lee et al. studied EEG signals from frontal and parietal scalp areas of human subjects under propofol anesthesia [42]. Effective connectivity was computed in both the awake and anesthetized states. The result showed that the anesthetized state greatly reduced feedback connectivity (frontal $\rightarrow$ parietal), indicating that higher order processing involving top-down selection and contextual interpretation of sensory information were impaired in anesthesia. In 2013, Lee et al. also demonstrated except propofol and sevoflurane, ketamine disrupt frontal-parietal communication, despite molecular and neurophysiologic differences [43]. Another EEG study also demonstrated impaired frontal to parietal, top-down information processing during anesthesia [44]. Analysis of effective connectivity in frontal-parietal pathway could provide a common metric of general anesthesia and insight into the cognitive neuroscience of anesthetic-induced unconsciousness.

In order to overcome methodological limitations of EEG methods, Jordan et al. recorded simultaneously resting-state EEG and blood oxygen level-dependent fMRI of healthy subjects during consciousness and propo- 
fol-induced LOC [45]. They found that changes in functional connectivity in fMRI during unconsciousness correlate with simultaneous changes in effective connectivity in EEG. Their work indicates that one underlying mechanism of functional decoupling of "higher-order" frontal and "lower-order" parietal processes during unconsciousness is the breakdown of directional feedback connectivity.

3) Posterior cingulate cortex

A third brain region that has been consistently implicated in studies examining activity modulations in anesthesia is the posterior cingulate cortex (PCC). Laureys and others reported diminished cerebral glucose metabolism by PET in PCC and noted impaired connectivity between PCC and other cortical areas in the anesthetized state when compared to controls [46]. But Stamatakis reported that increasing plasma propofol concentrations did not significantly modulate connectivity within posterior cingulate, the medial prefrontal cortex and bilateral parietal cortices. On the contrary, it was associated with significant increases in connectivity between the PCC and other areas (such as the motor/somatosensory cortices, the anterior thalamus, and the pontine tegmentum in the brainstem [47].

How is it possible to reconcile these two findings of PCC? The first reason is the difference of anesthesia. Laureys researched the patient who was in coma after suicide attempt using insulin and benzodiazepines, while Stamatakis used propofol on patients. The second argument point to a potentially more fundamental difference is physiological signals used for constructing connectivity. Laureys utilized PETs, and Stamatakis made use of fMRI.

\section{Studying Mechanisms of Postoperative Cognitive Dysfunction through Effective Connectivity}

Postoperative cognitive dysfunction (POCD) is now recognized as one such complication, often detected by an abnormality on neuropsychological testing [48]-[50]. It may manifest clinically as memory loss, psychomotor derangement, dementia, delirium or depression, difficulties with fine-motor coordination and impaired higherlevel cognitive functions [51] [52].

Working memory refers to the temporary storage and manipulation of information necessary for performance of complex cognitive tasks [53] [54]. Prefrontal cortex participates in working memory, and plays an active role in the shift of attention and task switching [55] [56]. Xu and others had researched the effect of propofol anesthesia on the working memory through gamma band LFPs in rat prefrontal cortex [57]. The result showed that prominent inhibition of working memory function was induced by propofol anesthesia, which mainly inhibited the effective connectivity of gamma-band LFPs in prefrontal cortex.

In 2013, Chi and colleagues found that postoperative pain contributes to the development of memory deficits after anesthesia and surgery via up-regulation of hippocampal NMDA receptors in aged rats [58]. Further, the synaptic NMDA receptor 2B level was reduced in the medial prefrontal cortex of adult mice suffering from surgical pain, which might contribute to POCD [59]. These findings suggest that postoperative pain may be one of important factor leading to POCD. In order to find out the mechanism that pain leads to POCD, Cardoso-Cruz H and others recorded LFPs from rat medial prefrontal cortex and dorsal hippocampus during a food-reinforced spatial working memory task, before and after the establishment of the spared nerve injury model of neuropathy [60]. The results show that the nerve lesion caused an impairment of working memory performance and revealed that chronic pain reduces the overall amount of information flowing in the prefrontal cortex-hippocampal circuit. Cardoso-Cruz's work demonstrates that effective connectivity disturbances in the prefrontal cortex-hippocampal circuit are a relevant cause for pain-related working memory deficits. Based on the above work, Cardoso-Cruz also researched the effective connectivity between prefrontal cortex and the mediodorsal thalamus through the same method [61]. The most relevant finding of his study is that the onset of chronic pain caused a global decrease in prefrontal cortex and the mediodorsal thalamus effective connectivity, and the decrease occurs over a wide frequency range and in both directions of the circuit, albeit more evidently from mediodorsal thalamus to prefrontal cortex.

\section{Effective Connectivity and Intrinsic Brain Activity}

\subsection{Hippocampus, Prefrontal Cortex, Hippocampus-Prefrontal Cortex}

The involvement of the hippocampus and the prefrontal cortex in cognitive processes and particularly in learning and memory has been known for a long time. There exists of a direct monosynaptic pathway from the ven- 
tral CA1 region of the hippocampus to prefrontal cortex in anatomy [62]. However, the medial prefrontal cortex, in rats and other species, has no direct return projections to the hippocampus, and few projections to parahippocampal structures including the entorhinal cortex. At present, the nucleus reuniens is well established to represent a critical link from the medial prefrontal cortex to the hippocampus [63].

In 2001, Fell and others showed effective connectivity between the hippocampus and the neocortex accompanies successful memory encoding [64]. Based on Fell work, Jones et al. disclosed theta frequency coordination of LFP between hippocampus and mPFC during behavioral epochs was presumed to construct effective communication between these two structures [65].

In 2007, Baeg and others demonstrated that the hippocampus-neocortex network is a primary conduit for cerebral information flow for learning and memory [66]. Then Adhikari et al. constructed effective connectivity between ventral hippocampus and prefrontal cortex during rat working memory task, and demonstrate that the ventral hippocampus leads the prefrontal cortex in the theta-frequency range [67]. Interestingly, under anesthesia, Taxidis and others had demonstrated that there exists a continuous information flow from hippocampus towards prefrontal cortex [68]. How to explain the results? After training, Rats in Adhikari work had formed long-time memory, which was mainly consolidated in hippocampus [69]. So information in the hippocampus-neocortex network flowed from hippocampus to prefrontal cortex during rat working memory task. There are more densely anesthetic receptors located in the neocortex than in the hippocampus, and the prefrontal cortex is inhibited by anesthetic preferentially in the neocortex than in the hippocampus [70]-[72]. As to Taxidis work, information in the hippocampus-neocortex network flowed from hippocampus to prefrontal cortex during rat anesthesia.

Based on the above work, information flows between hippocampus and prefrontal cortex is fundamental for learning and memory, and disruption of such communication is likely to generate behavioral impairments.

\subsection{Cortex and the Striatum}

Nakhnikian and others characterized directed influence between cortex and striatum using LFPs recorded in primary motor cortex and dorsal striatum from awake, unrestrained rats [73]. In addition to observing free behaviors, they recorded during sleep and anesthesia in order to compare rest states to spontaneous behavior and to each other, and to assess the neural correlates of drug induced and natural inactivity. They found that effective connectivity became bidirectional in freely behaving animals. In agreement with Sharott A report, effective connectivity was unidirectional, from cortex to striatum, during natural sleep and anesthesia [74].

\subsection{Amygdale and Emotion}

Alkire et al. used structural equation modeling of the PET data revealing that anesthesia suppressed amygdala to hippocampal effective connectivity [75]. The behavioral results show that anesthesia blocks emotional memory, and connectivity results demonstrate that anesthesia suppresses the effective influence of the amygdala. Collectively, the findings support the hypothesis that the amygdale mediates memory modulation by demonstrating that suppressed amygdala effectiveness equates with a loss of emotional memory.

\section{The Continued Study of Effective Connectivity in Anesthesia}

In discussing the effective connectivity of the brain and its application in anesthesia issue, we have come across a number of issues that can and should be addressed in future studies.

1) Dosage of anesthesia

Although anesthesia has the LOC as a potential endpoint, there are many levels of anesthesia, all of which have potential relevance to the study of effective connectivity in the brain.

Most studies of effective connectivity in the anesthetized state have reported the anesthesia by the standard of minimum alveolar concentration (MAC) or a behavioral endpoint. Although both of the methods are useful, there is one obstacle hindering comparisons across studies. For example, the induction of one kind anesthesia in one subject may not be complete at one dosage, whereas it may be in another subject. The key point may leave out critical information for the interpretation of that study.

2) Type of anesthetic

Although it may seem that the endpoint of surgical anesthesia is sufficiently similar across anesthetics, this cannot and should not be assumed in effective connectivity studies, because there is not a common effective 
connectivity similar across anesthetic types. So, it is important to continue to assess effective connectivity across a variety of anesthetic types, at least consisting of a single representative of three or four major classes of anesthetics.

3) Method of assessing effective connectivity

Although many methods have offered the ability to assess effective connectivity, each method has its strengths and weaknesses. GC and methods based on GC (such as DTF and PDC) remain the most heavily used methods of computing effective connectivity relations. Regardless of the methodology used, however, the need for effective connectivity studies across anesthesia and consciousness state remains significant, as they provide unique insight into both potential mechanisms of anesthesia and intrinsic functional activity in the brain.

\section{Acknowledgements}

This work was supported by the National Natural Science Foundation of China (NSFC 61375113, 61301252 and 81371245), Science and Technology Supported Key Project of Tianjin (12ZCZDSY03000), China Postdoctoral Science Foundation funded project (2014M561193), the funders had no role in study design, data collection and analysis, decision to publish, or preparation of the manuscript.

\section{Conflict of Interests}

The authors have declared that no conflict of interests exists.

\section{References}

[1] Ramón y Cajal, S. (1995) Histology of the Nervous System of Man and Vertebrates. Oxford University Press, Oxford.

[2] Swanson, L.W. (2003) Brain Architecture: Understanding the Basic Plan. Oxford University Press, Oxford.

[3] Bressler, S.L. (1995) Large-Scale Cortical Networks and Cognition. Brain Research Reviews, 20, $288-304$. http://dx.doi.org/10.1016/0165-0173(94)00016-I

[4] Buzsáki, G. (2006) Rhythms of the Brain. Oxford University Press, Oxford.

[5] McIntosh, A.R. (2000) Towards a Network Theory of Cognition. Neural Networks, 13, 861-870.

[6] Mesulam, M.M. (1998) From Sensation to Cognition. Brain, 121, 1013-1052.

[7] Feldt, S., Bonifazi, P. and Cossart, R. (2011) Dissecting Functional Connectivity of Neuronal Microcircuits: Experimental and Theoretical Insights. Trends in Neurosciences, 34, 225-236. http://dx.doi.org/10.1016/j.tins.2011.02.007

[8] Bullmore, E. and Sporns, O. (2009) Complex Brain Networks: Graph Theoretical Analysis of Structural and Functional Systems. Nature Reviews Neuroscience, 10, 186-198. http://dx.doi.org/10.1038/nrn2575

[9] Friston, K.J., Harrison, L. and Penny, W. (2003) Dynamic Causal Modelling. NeuroImage, 19, 1273-1302. http://dx.doi.org/10.1016/S1053-8119(03)00202-7

[10] Rodrigues, J. and Andrade, A. (2014) Lag-Based Effective Connectivity Applied to fMRI: A Simulation Study Highlighting Dependence on Experimental Parameters and Formulation. NeuroImage, 89, 358-377. http://dx.doi.org/10.1016/j.neuroimage.2013.10.029

[11] Barrett, A.B., Barnett, L. and Seth, A.K. (2010) Multivariate Granger Causality and Generalized Variance. Physical Review E: Statistical, Nonlinear, and Soft Matter Physics, 81, Article ID: 041907. http://dx.doi.org/10.1103/PhysRevE.81.041907

[12] Barrett, A.B., Murphy, M., Bruno, M.A., Noirhomme, Q., Boly, M., Laureys, S., et al. (2012) Granger Causality Analysis of Steady-State Electroencephalographic Signals during Propofol-Induced Anaesthesia. PLoS ONE, 7, e29072. http://dx.doi.org/10.1371/journal.pone.0029072

[13] Lu, Q., Bi, K., Liu, C., Luo, G.P., Tang, H. and Yao, Z.J. (2013) Predicting Depression Based on Dynamic Regional Connectivity: A Windowed Granger Causality Analysis of MEG Recordings. Brain Research, 1535, 52-60. http://dx.doi.org/10.1016/j.brainres.2013.08.033

[14] Zhang, L., Chen, G.F., Niu, R.F., Wei, W., Ma, X.Y., Xu, J.M., et al. (2012) Hippocampal Theta-Driving Cells Revealed by Granger Causality. Hippocampus, 22, 1781-1793. http://dx.doi.org/10.1002/hipo.22012

[15] David, O., Guillemain, I., Saillet, S., Reyt, S., Deransart, C., Segebarth, C., et al. (2008) Identifying Neural Drivers with Functional MRI: An Electrophysiological Validation. PLoS Biology, 6, 2683-2697. http://dx.doi.org/10.1371/journal.pbio.0060315

[16] Roebroeck, A., Formisano, E. and Goebel, R. (2005) Mapping Directed Influence over the Brain Using Granger Cau- 
sality and fMRI. NeuroImage, 25, 230-242. http://dx.doi.org/10.1016/j.neuroimage.2004.11.017

[17] Kaminski, M., Ding, M.Z., Truccolo, W.A. and Bressler, S.L. (2001) Evaluating Causal Relations in Neural Systems: Granger Causality, Directed Transfer Function and Statistical Assessment of Significance. Biological Cybernetics, 85, 145-157. http://dx.doi.org/10.1007/s004220000235

[18] Baccala, L.A. and Sameshima, K. (2001) Partial Directed Coherence: A New Concept in Neural Structure Determination. Biological Cybernetics, 84, 463-474. http://dx.doi.org/10.1007/PL00007990

[19] Eichler, M. (2006) On the Evaluation of Information Flow in Multivariate Systems by the Directed Transfer Function. Biological Cybernetics, 94, 469-482. http://dx.doi.org/10.1007/s00422-006-0062-z

[20] Schelter, B., Winterhalder, M., Eichler, M., Peifer, M., Hellwig, B., Guschlbauer, B., et al. (2006) Testing for Directed Influences among Neural Signals Using Partial Directed Coherence. Journal of Neuroscience Methods, 152, $210-219$. http://dx.doi.org/10.1016/i.jneumeth.2005.09.001

[21] Nolte, G., Ziehe, A., Nikulin, V.V., Schlög, A., Kräme, N., Brismar, T., et al. (2008) Robustly Estimating the Flow Direction of Information in Complex Physical Systems. Physical Review Letters, 100, Article ID: 234101.

http://dx.doi.org/10.1103/PhysRevLett.100.234101

[22] Schreiber, T. (2000) Measuring Information Transfer. Physical Review Letters, 85, 461. http://dx.doi.org/10.1103/PhysRevLett.85.461

[23] Chávez, M., Le Van Quyen, M., Navarro, V., Baulac, M. and Martinerie, J. (2003) Spatio-Temporal Dynamics Prior to Neocortical Seizures: Amplitude versus Phase Couplings. IEEE Transactions on Biomedical Engineering, 50, 571-583. http://dx.doi.org/10.1109/TBME.2003.810696

[24] Paluš, M. and Stefanovska, A. (2003) Direction of Coupling from Phases of Interacting Oscillators: An InformationTheoretic Approach. Physical Review E: Statistical, Nonlinear, and Soft Matter Physics, 67, Article ID: 055201. http://dx.doi.org/10.1103/PhysRevE.67.055201

[25] Alkire, M.T., Haier, R.J. and Fallon, J.H. (2000) Toward a Unified Theory of Narcosis: Brain Imaging Evidence for a Thalamocortical Switch as the Neurophysiologic Basis of Anesthetic-Induced Unconsciousness. Consciousness and Cognition, 9, 370-386. http://dx.doi.org/10.1006/ccog.1999.0423

[26] Cariani, P. (2000) Anesthesia, Neural Information Processing, and Conscious Awareness. Consciousness and Cognition, 9, 387-395. http://dx.doi.org/10.1006/ccog.1999.0420

[27] Alkire, M.T. and Miller, J. (2005) General Anesthesia and the Neural Correlates of Consciousness. Progress in Brain Research, 150, 229-244, 596-597. http://dx.doi.org/10.1016/S0079-6123(05)50017-7

[28] Newman, J. (1995) Thalamic Contributions to Attention and Consciousness. Consciousness and Cognition, 4, $172-193$. http://dx.doi.org/10.1006/ccog.1995.1024

[29] Angel, A. (1993) Central Neuronal Pathways and the Process of Anaesthesia. British Journal of Anaesthesia, 71, 148163. http://dx.doi.org/10.1093/bja/71.1.148

[30] Volkow, N.D., Wang, G.J., Hitzemann, R., Fowler, J.S., Pappas, N., Lowrimore, P., et al. (1995) Depression of Thalamic Metabolism by Lorazepam Is Associated with Sleepiness. Neuropsychopharmacology, 12, 123-132. http://dx.doi.org/10.1016/0893-133X(94)00068-B

[31] Alkire, M.T., Pomfrett, C.J., Haier, R.J., Gianzero, M.V., Chan, C.M., Jacobsen, B.P., et al. (1999) Functional Brain Imaging during Anesthesia in Humans: Effects of Halothane on Global and Regional Cerebral Glucose Metabolism. Anesthesiology, 90, 701-709. http://dx.doi.org/10.1097/00000542-199903000-00011

[32] Fiset, P., Paus, T., Daloze, T., Plourde, G., Meuret, P., Bonhomme, V., et al. (1999) Brain Mechanisms of PropofolInduced Loss of Consciousness in Humans: A Positron Emission Tomographic Study. The Journal of Neuroscience, 19, 5506-5513.

[33] Alkire, M.T., Hudetz, A.G. and Tononi, G. (2008) Consciousness and Anesthesia. Science, 322, 876-880. http://dx.doi.org/10.1126/science.1149213

[34] Ward, L.M. (2011) The Thalamic Dynamic Core Theory of Conscious Experience. Consciousness and Cognition, 20, 464-486. http://dx.doi.org/10.1016/j.concog.2011.01.007

[35] Andrada, J., Livingston, P., Lee, B.J. and Antognini, J. (2012) Propofol and Etomidate Depress Cortical, Thalamic, and Reticular Formation Neurons during Anesthetic-Induced Unconsciousness. Anesthesia and Analgesia, 114, 661-669. http://dx.doi.org/10.1213/ANE.0b013e3182405228

[36] Kim, S.P., Hwang, E., Kang, J.H., Kim, S. and Choi, J.H. (2012) Changes in the Thalamocortical Connectivity during Anesthesia-Induced Transitions in Consciousness. Neuroreport, 23, 294-298. http://dx.doi.org/10.1097/WNR.0b013e3283509ba0

[37] Velly, L.J., Rey, M.F., Bruder, N.J., Gouvitsos, F.A., Witjas, T., Regis, J.M., et al. (2007) Differential Dynamic of Action on Cortical and Subcortical Structures of Anesthetic Agents during Induction of Anesthesia. Anesthesiology, 107, 
202-212. http://dx.doi.org/10.1097/01.anes.0000270734.99298.b4

[38] Naghavi, H.R. and Nyberg, L. (2005) Common Fronto-Parietal Activity in Attention, Memory, and Consciousness: Shared Demands on Integration? Consciousness and Cognition, 14, 390-425. http://dx.doi.org/10.1016/j.concog.2004.10.003

[39] Rees, G., Kreiman, G. and Koch, C. (2002) Neural Correlates of Consciousness in Humans. Nature Reviews Neuroscience, 3, 261-270. http://dx.doi.org/10.1038/nrn783

[40] Sarter, M., Givens, B. and Bruno, J.P. (2001) The Cognitive Neuroscience of Sustained Attention: Where Top-Down Meets Bottom-Up. Brain Research Brain Research Reviews, 35, 146-160. http://dx.doi.org/10.1038/nrn783

[41] Imas, O.A., Ropella, K.M., Ward, B.D., Wood, J.D. and Hudetz, A.G. (2005) Volatile Anesthetics Disrupt FrontalPosterior Recurrent Information Transfer at Gamma Frequencies in Rat. Neuroscience Letters, 387, 145-150. http://dx.doi.org/10.1016/j.neulet.2005.06.018

[42] Lee, U.C., Kim, S., Noh, G.J., Choi, B.M., Hwang, E. and Mashour, G.A. (2009) The Directionality and Functional Organization of Frontoparietal Connectivity during Consciousness and Anesthesia in Humans. Consciousness and Cognition, 18, 1069-1078. http://dx.doi.org/10.1016/j.concog.2009.04.004

[43] Lee, U.C., Ku, S.W., Noh, G., Baek, S., Choi, B. and Mashour, G.A. (2013) Disruption of Frontal-Parietal Communication by Ketamine, Propofol, and Sevoflurane. Anesthesiology, 118, 1264-1275. http://dx.doi.org/10.1097/ALN.0b013e31829103f5

[44] Ku, S.W., Lee, U.C., Noh, G.J., Jun, I.G. and Mashour, G.A. (2011) Preferential Inhibition of Frontal-to-Parietal Feedback Connectivity Is a Neurophysiologic Correlate of General Anesthesia in Surgical Patients. PLoS ONE, 6, e25155. http://dx.doi.org/10.1371/journal.pone.0025155

[45] Jordan, D., Ilg, R., Riedl, V., Schorer, A., Grimberg, S., Neufang, S., et al. (2013) Simultaneous Electroencephalographic and Functional Magnetic Resonance Imaging Indicate Impaired Cortical Top-Down Processing in Association with Anesthetic-Induced Unconsciousness. Anesthesiology, 119, 1031-1042. http://dx.doi.org/10.1097/ALN.0b013e3182a7ca92

[46] Laureys, S., Goldman, S., Phillips, C., Van Bogaert, P., Aerts, J., Luxen, A., et al. (1999) Impaired Effective Cortical Connectivity in Vegetative State: Preliminary Investigation Using PET. NeuroImage, 9, 377-382. http://dx.doi.org/10.1006/nimg.1998.0414

[47] Stamatakis, E.A., Adapa, R.M., Absalom, A.R. and Menon, D.K. (2010) Changes in Resting Neural Connectivity during Propofol Sedation. PLoS ONE, 5, e14224. http://dx.doi.org/10.1371/journal.pone.0014224

[48] Moller, J.T., Cluitmans, P., Rasmussen, L.S., Houx, P., Rasmussen, H., Canet, J., et al. (1998) Long-Term Postoperative Cognitive Dysfunction in the Elderly ISPOCD1 Study. The Lancet, 351, 857-861. http://dx.doi.org/10.1016/S0140-6736(97)07382-0

[49] Mrak, R.E., Griffin, S.T. and Graham, D.I. (1997) Aging-Associated Changes in Human Brain. Journal of Neuropathology and Experimental Neurology, 56, 1269-1275. http://dx.doi.org/10.1097/00005072-199712000-00001

[50] Seymour, D.G. and Severn, A.M. (2009) Cognitive Dysfunction after Surgery and Anaesthesia: What Can We Tell the Grandparents? Age and Ageing, 38, 147-150. http://dx.doi.org/10.1093/ageing/afn289

[51] Butterfield, N.N., Graf, P., Ries, C.R. and MacLeod, B.A. (2004) The Effect of Repeated Isoflurane Anesthesia on Spatial and Psychomotor Performance in Young and Aged Mice. Anesthesia and Analgesia, 98, 1305-1311. http://dx.doi.org/10.1213/01.ANE.0000108484.91089.13

[52] Raja, P.V., Blumenthal, J.A. and Doraiswamy, P.M. (2004) Cognitive Deficits Following Coronary Artery Bypass Grafting: Prevalence, Prognosis, and Therapeutic Strategies. CNS Spectrums, 9, 763-772.

[53] Baddeley, A. (1992) Working Memory. Science, 255, 556-559. http://dx.doi.org/10.1126/science.1736359

[54] Baddeley, A. (2003) Working Memory: Looking Back and Looking Forward. Nature Reviews Neuroscience, 4, 829839. http://dx.doi.org/10.1038/nrn1201

[55] Hyafil, A., Summerfield, C. and Koechlin, E. (2009) Two Mechanisms for Task Switching in the Prefrontal Cortex. The Journal of Neuroscience, 29, 5135-5142. http://dx.doi.org/10.1523/JNEUROSCI.2828-08.2009

[56] Rossi, A.F., Pessoa, L., Desimone, R. and Ungerleider, L.G. (2009) The Prefrontal Cortex and the Executive Control of Attention. Experimental Brain Research, 192, 489-497. http://dx.doi.org/10.1007/s00221-008-1642-Z

[57] Xu, X.Y., Tian, Y., Li, S.Y., Li, Y.Z., Wang, G.L. and Tian, X. (2013) Inhibition of Propofol Anesthesia on Functional Connectivity between LFPs in PFC during Rat Working Memory Task. PLoS ONE, 8, e83653. http://dx.doi.org/10.1371/journal.pone.0083653

[58] Chi, H.D., Kawano, T., Tamura, T., Iwata, H., Takahashi, Y., Eguchi, S., et al. (2013) Postoperative Pain Impairs Subsequent Performance on a Spatial Memory Task via Effects on N-methyl-D-Aspartate Receptor in Aged Rats. Life Sciences, 93, 986-993. http://dx.doi.org/10.1016/j.1fs.2013.10.028 
[59] Zhang, X.Q., Xin, X., Dong, Y.L., Zhang, Y.Y., Yu, B.W., Mao, J.R., et al. (2013) Surgical Incision-Induced Nociception Causes Cognitive Impairment and Reduction in Synaptic NMDA Receptor 2B in Mice. The Journal of Neuroscience, 33, 17737-17748. http://dx.doi.org/10.1523/JNEUROSCI.2049-13.2013

[60] Cardoso-Cruz, H., Lima, D. and Galhardo, V. (2013) Impaired Spatial Memory Performance in a Rat Model of Neuropathic Pain Is Associated with Reduced Hippocampus-Prefrontal Cortex Connectivity. The Journal of Neuroscience, 33, 2465-2480. http://dx.doi.org/10.1523/JNEUROSCI.5197-12.2013

[61] Cardoso-Cruz, H., Sousa, M., Vieira, J.B., Lima, D. and Galhardo, V. (2013) Prefrontal Cortex and Mediodorsal Thalamus Reduced Connectivity Is Associated with Spatial Working Memory Impairment in Rats with Inflammatory Pain. Pain, 154, 2397-2406. http://dx.doi.org/10.1016/j.pain.2013.07.020

[62] Laroche, S., Davis, S. and Jay, T.M. (2000) Plasticity at Hippocampal to Prefrontal Cortex Synapses: Dual Roles in Working Memory and Consolidation. Hippocampus, 10, 438-446. http://dx.doi.org/10.1002/1098-1063(2000)10:4<438::AID-HIPO10>3.0.CO;2-3

[63] Vertes, R.P., Hoover, W.B., Szigeti-Buck, K. and Leranth, C. (2007) Nucleus Reuniens of the Midline Thalamus: Link between the Medial Prefrontal Cortex and the Hippocampus. Brain Research Bulletin, 71, 601-609. http://dx.doi.org/10.1016/j.brainresbull.2006.12.002

[64] Fell, J., Klaver, P., Lehnertz, K., Grunwald, T., Schaller, C., Elger, C.E., et al. (2001) Human Memory Formation Is Accompanied by Rhinal-Hippocampal Coupling and Decoupling. Nature Neuroscience, 4, 1259-1264. http://dx.doi.org/10.1038/nn759

[65] Jones, M.W. and Wilson, M.A. (2005) Theta Rhythms Coordinate Hippocampal-Prefrontal Interactions in a Spatial Memory Task. PLoS Biology, 3, e402. http://dx.doi.org/10.1371/journal.pbio.0030402

[66] Baeg, E.H., Kim, Y.B., Kim, J., Ghim, J.W., Kim, J.J. and Jung, M.W. (2007) Learning-Induced Enduring Changes in Functional Connectivity among Prefrontal Cortical Neurons. The Journal of Neuroscience, 27, 909-918. http://dx.doi.org/10.1523/JNEUROSCI.4759-06.2007

[67] Adhikari, A., Sigurdsson, T., Topiwala, M.A. and Gordon, J.A. (2010) Cross-Correlation of Instantaneous Amplitudes of Field Potential Oscillations: A Straightforward Method to Estimate the Directionality and Lag between Brain Areas. Journal of Neuroscience Methods, 191, 191-200. http://dx.doi.org/10.1016/j.jneumeth.2010.06.019

[68] Taxidis, J., Coomber, B., Mason, R. and Owen, M. (2010) Assessing Cortico-Hippocampal Functional Connectivity under Anesthesia and Kainic Acid Using Generalized Partial Directed Coherence. Biological Cybernetics, 102, 327340. http://dx.doi.org/10.1007/s00422-010-0370-1

[69] Brockmann, M.D., Pöschel, B., Cichon, N. and Hanganu-Opatz, I.L. (2011) Coupled Oscillations Mediate Directed Interactions between Prefrontal Cortex and Hippocampus of the Neonatal Rat. Neuron, 71, 332-347. http://dx.doi.org/10.1016/j.neuron.2011.05.041

[70] Hentschke, H., Schwarz, C. and Antkowiak, B. (2005) Neocortex Is the Major Target of Sedative Concentrations of Volatile Anaesthetics: Strong Depression of Firing Rates and Increase of GABAA Receptor-Mediated Inhibition. The European Journal of Neuroscience, 21, 93-102. http://dx.doi.org/10.1111/j.1460-9568.2004.03843.x

[71] Kaisti, K.K., Metsahonkala, L., Teräs, M., Oikonen, V., Aalto, S., Jääskeläinen, S., et al. (2002) Effects of Surgical Levels of Propofol and Sevoflurane Anesthesia on Cerebral Blood Flow in Healthy Subjects Studied with Positron Emission Tomography. Anesthesiology, 96, 1358-1370. http://dx.doi.org/10.1097/00000542-200206000-00015

[72] Solt, K. and Forman, S.A. (2007) Correlating the Clinical Actions and Molecular Mechanisms of General Anesthetics. Current Opinion in Anaesthesiology, 20, 300-306. http://dx.doi.org/10.1097/ACO.0b013e32816678a5

[73] Nakhnikian, A., Rebec, G.V., Grasse, L.M., Dwiel, L.L., Shimono, M. and Beggs, J.M. (2014) Behavior Modulates Effective Connectivity between Cortex and Striatum. PLoS ONE, 9, e89443. http://dx.doi.org/10.1371/journal.pone.0089443

[74] Sharott, A., Magill, P.J., Bolam, J.P. and Brown, P. (2005) Directional Analysis of Coherent Oscillatory Field Potentials in the Cerebral Cortex and Basal Ganglia of the Rat. The Journal of Physiology, 562, 951-963. http://dx.doi.org/10.1113/jphysiol.2004.073189

[75] Alkire, M.T., Gruver, R., Miller, J., McReynolds, J.R., Hahn, E.L. and Cahill, L. (2008) Neuroimaging Analysis of an Anesthetic Gas That Blocks Human Emotional Memory. Proceedings of the National Academy of Sciences of the United States of America, 105, 1722-1727. http://dx.doi.org/10.1073/pnas.0711651105 